\title{
Substrate Interaction
}

National Cancer Institute

\section{Source}

National Cancer Institute. Substrate Interaction. NCI Thesaurus. Code C41556.

Substrate Interaction involves temporary non-covalent binding through intermolecular physical forces of attraction and spatial complementarity between biologically-active molecules and their target molecule or between a biological molecule and an underlaying surface. 